\title{
Large-Eddy Simulation of the Lid-Driven Cubic Cavity Flow by the Spectral Element Method
}

\author{
Roland Bouffanais, ${ }^{1}$ Michel O. Deville, ${ }^{1}$ Paul F. Fischer, ${ }^{2}$ \\ Emmanuel Leriche, ${ }^{1}$ and Daniel Weill ${ }^{1}$
}

Received October 14, 2004; accepted (in revised form) August 23, 2005; Published online March 13, 2006

\begin{abstract}
This paper presents the large-eddy simulation of the lid-driven cubic cavity flow by the spectral element method (SEM) using the dynamic model. Two spectral filtering techniques suitable for these simulations have been implemented. Numerical results for Reynolds number $\mathrm{Re}=12,000$ are showing very good agreement with other experimental and DNS results found in the literature.
\end{abstract}

KEY WORDS: Spectral element; spectral filter; LES; lid-driven cavity.

\section{INTRODUCTION}

Spectral element methods (SEM) have been mainly applied to the direct numerical simulation (DNS) of fluid flow problems at low and moderate Reynolds (Re) numbers. With the advent of more powerful computers, especially through cluster technology, higher Re values seem to fall within the realm of feasibility. However, despite their high accuracy, SEM are still far from reaching industrial applications that involve developed turbulence at $\mathrm{Re}$ values of the order of $10^{6}-10^{7}$. The reason for that dismal performance is that a resolved DNS including all scales from the gross structures to Kolmogorov scales, needs a number of degrees of freedom (dof) that grows like $\mathrm{Re}^{9 / 4}$. Therefore with increasing Re, we have to increase the number of elements, $E$, and the degree, $N$, of the polynomial spaces. This places the computational load far out of the reach of present day computers.

\footnotetext{
${ }^{1}$ Laboratory of Computational Engineering, Ecole Polytechnique Fédérale de Lausanne, CH-1015 Lausanne, Switzerland. E-mail: Michel.Deville@epfl.ch

${ }^{2}$ MCS, Argonne National Laboratory, 9700 S. Cass Avenue, Argonne, IL 60439, USA.
} 
Large-eddy simulation (LES) represents an alternative to DNS insofar that it involves less dofs because the behaviour of the small scales are modeled. The LES methodology deals with high Re number unsteady flows by using coarse meshes in which the contributions of small subgrid scale (SGS) structures are modeled while the large-scale structures are obtained by the computed flow dynamics. The reader is referred to the monograph by Sagaut [15] for details. In this paper we will focus our attention on the dynamic model (DM) $[4,11,15]$ which will be evaluated by comparing LES numerical results and DNS on the three-dimensional cubic cavity flow.

\section{MATHEMATICAL MODELLING}

\subsection{The Governing Equations}

Large-scale quantities, designated by an "overbar", are obtained by a filtering process on the domain $\Omega$. Assuming the filter commutes with differentiation and applying the filter to the Navier-Stokes equations in divergence form for the non-linear term, one obtains the following relations:

$$
\begin{aligned}
\frac{\partial \overline{\mathbf{v}}}{\partial t}+\nabla \cdot \overline{\mathbf{v}} \overline{\mathbf{v}} & =-\nabla \bar{p}+\nu \boldsymbol{\Delta} \overline{\mathbf{v}}-\nabla \cdot \boldsymbol{\tau}, \\
\nabla \cdot \overline{\mathbf{v}} & =0 .
\end{aligned}
$$

Here, $\overline{\mathbf{v}}$ is the filtered velocity, $t$ denotes the time, $\bar{p}$ is the filtered pressure divided by the constant density, $v$ the kinematic viscosity. The symbols $\nabla$ and $\Delta$ represent the nabla and Laplacian operators, respectively. The SGS stress tensor $\tau$ takes the small-scale effects into account and is given by

$$
\tau=\overline{\mathbf{v}}-\overline{\mathbf{v}} \overline{\mathbf{v}} \text {. }
$$

\subsection{Smagorinksy and Dynamic Models}

The SGS Smagorinsky model (SM) [17] uses the concept of turbulent viscosity and assumes that the small scales are in equilibrium, balancing energy production and dissipation. This yields the following expression for the eddy-viscosity

$$
v_{T}=\left(C_{\mathrm{S}} \bar{\Delta}\right)^{2}|\overline{\mathbf{S}}|,
$$

where $|\overline{\mathbf{S}}|=\left(2 \bar{S}_{i j} \bar{S}_{i j}\right)^{1 / 2}$ is the magnitude filtered strain-rate-tensor, $C_{\mathrm{S}}$ is the Smagorinsky constant and $\bar{\Delta}$ the filter width. The SM has several 
drawbacks. The most severe one is the constant value of $C_{\mathrm{S}}$ during the computation which produces too much dissipation. Furthermore the SM does not provide the modeller with backscattering where kinetic energy is transferred from small scales to larger scales.

The dynamic model proposed by Germano et al. [4] overcomes the difficulty of constant $C_{\mathrm{S}}$, by allowing it to become dependent of space and time. Now we have a dynamic constant $C_{\mathrm{d}}=C_{\mathrm{d}}(\mathbf{x}, t)$. Let us introduce a test-filter length scale $\hat{\Delta}$ that is larger than the grid length scale $\bar{\Delta}$ (e.g. $\hat{\Delta}=2 \bar{\Delta}$ ). Using the information provided by those two filters and assuming that in the inertial range of the turbulence energy spectrum, the statistical self-similarity applies, we can better determine the features of the SGS stress. With the test filter, the former LES Eq. (1) yield a relation involving the sub-test-scale stress

$$
\mathbf{T}=\hat{\overline{\mathbf{v}}}-\hat{\mathbf{v}} \hat{\overline{\mathbf{v}}} .
$$

We introduce the Germano identity to obtain the relation between $\mathbf{T}$ and the filtered $\hat{\boldsymbol{\tau}}$ such that

$$
\mathbf{L}=\mathbf{T}-\hat{\boldsymbol{\tau}}=\widehat{\mathbf{v}} \overline{\mathbf{v}}-\hat{\mathbf{v}} \hat{\overline{\mathbf{v}}}
$$

We apply the eddy viscosity model to $\boldsymbol{\tau}$ and $\mathbf{T}$ and we obtain using the self-similarity hypothesis for the constant $C_{\mathrm{d}}$

$$
\boldsymbol{\tau}-\frac{1}{3} \operatorname{tr}(\boldsymbol{\tau}) \mathbf{I}=-2 C_{\mathrm{d}} \overline{\boldsymbol{\Delta}}^{2}|\overline{\mathbf{S}}| \overline{\mathbf{S}}=C_{\mathrm{d}} \boldsymbol{\beta},
$$

and

$$
\mathbf{T}-\frac{1}{3} \operatorname{tr}(\mathbf{T}) \mathbf{I}=-2 C_{\mathrm{d}} \hat{\overline{\boldsymbol{\Delta}}}^{2}|\hat{\mathbf{S}}| \hat{\mathbf{S}}=C_{\mathrm{d}} \boldsymbol{\alpha},
$$

where the symbol $t r$ denotes the trace of the tensor. Inserting (7) and (8) in the deviatoric part $\mathbf{L}^{\mathrm{d}}$ of $\mathbf{L}$ produces

$$
\mathbf{L}-\frac{1}{3} \operatorname{tr}(\mathbf{L}) \mathbf{I} \equiv \mathbf{L}^{\mathrm{d}}=C_{\mathrm{d}} \boldsymbol{\alpha}-\widehat{C_{\mathrm{d}} \boldsymbol{\beta}} .
$$

Assuming that $C_{\mathrm{d}}$ does not vary too much in space, one sets $\widehat{C}_{\mathrm{d}} \approx C_{\mathrm{d}}$ and one can deduce from a least square minimization of the error related to (9) (see $[12,15])$ that

$$
C_{\mathrm{d}}=\frac{(\boldsymbol{\alpha}-\widehat{\boldsymbol{\beta}}): \mathbf{L}^{\mathrm{d}}}{(\boldsymbol{\alpha}-\widehat{\boldsymbol{\beta}}):(\boldsymbol{\alpha}-\widehat{\boldsymbol{\beta}})},
$$

where the notation : is used for inner tensor product (double contraction). 


\section{NUMERICAL APPROXIMATION}

\subsection{Space Discretization}

The numerical approximation is obtained through a weak formulation of the Eqs. (1)-(2) discretized using the Lagrange-Legendre approximation. The reader is referred to the monograph by Deville et al. [3] for details. The velocity and pressure are expressed in the $\mathbb{P}_{N}-\mathbb{P}_{N-2}$ spaces where $\mathbb{P}_{N}$ is the set of polynomials of degree $\leqslant N$ in each space direction. This approximation avoids the presence of spurious pressure modes as it was proved by Maday and Patera [13]. The quadrature rules involved in the weak formulation define a Gauss-Lobatto-Legendre (GLL) grid for the velocity nodes and a Gauss-Legendre grid (GL) for the nodal pressures.

Borrowing the notation from [3], the semi-discrete filtered NavierStokes equations resulting from the spectral element discretization are

$$
\begin{aligned}
\mathbf{M} \frac{\mathrm{d} \overline{\overline{\mathbf{v}}}}{\mathrm{d} t}+\mathbf{C} \underline{\overline{\mathbf{v}}}+v \mathbf{K} \underline{\overline{\mathbf{v}}}-\mathbf{D}^{T} \underline{\bar{p}}+\mathbf{D} \underline{\mathbf{\tau}}=0, \\
-\mathbf{D} \overline{\overline{\mathbf{v}}}=0 .
\end{aligned}
$$

The diagonal mass matrix $\mathbf{M}$ is composed of $d$ blocks, the mass matrices $M$, with $d=3$ for the three-dimensional cavity problem. The supervector $\overline{\mathbf{v}}$ contains all the nodal velocity components while $\bar{p}$ is made of all nodal pressures. The matrices $\mathbf{K}, \mathbf{D}^{T}, \mathbf{D}$ are the discrete Laplacian, gradient and divergence operators, respectively. The matrix operator $\mathbf{C}$ represents the action of the non-linear advection term written in convective form $\overline{\mathbf{v}} \cdot \nabla$, on the velocity field and depends on $\overline{\mathbf{v}}$ itself. The spatial discretization leads to a set of non-linear ordinary differential equations (11) subject to the incompressibility condition (12).

\subsection{Time Integration}

As the LES viscosity is not invariant, we modify the standard time integration scheme in such a way that this space varying viscosity be handled explicitly as this was done e.g. in $[1,6]$. Let us define the effective viscosity as

$$
v_{\mathrm{eff}}=v+v_{T}=v_{\mathrm{cst}}+\left(v_{\mathrm{eff}}-v_{\mathrm{cst}}\right),
$$

where $v_{\text {cst }}$ is the sum of $v$ and the spatial average of $v_{T}$ over the computational domain, being by construction constant in space but not in time. 
The filtered Navier-Stokes equations become

$$
\begin{aligned}
\mathbf{M} \frac{\mathrm{d} \overline{\underline{\mathbf{v}}}}{\mathrm{d} t}+v_{\mathrm{cst}} \mathbf{K} \underline{\overline{\mathbf{v}}}-\mathbf{D}^{T} \underline{\bar{p}} & =-\mathbf{C} \underline{\mathbf{v}}+2 \mathbf{D}\left(v_{\mathrm{eff}}-v_{\mathrm{cst}}\right) \underline{\overline{\mathbf{S}}}, \\
-\mathbf{D} \underline{\overline{\mathbf{v}}} & =0 .
\end{aligned}
$$

The viscous linear term and the pressure are implicitly integrated by a backward differentiation formula of order 2 (BDF2) while the terms in the right-hand side of Eq. (14) are computed by a second order extrapolation method (EX2). The explicit viscous part $2 \mathbf{D}\left(v_{\text {eff }}-v_{\text {cst }}\right) \underline{\overline{\mathbf{S}}}$ leads to a stability condition such that $\left(v_{\text {eff }}-v_{\text {cst }}\right) \Delta t \leqslant C / N^{4}$ while the CFL condition restricts the time step such that $\bar{u}_{\max } \Delta t \leqslant C / N^{2}$. It would seem that the viscous restriction is more severe than the convective one. However the magnitude of the term $2 \mathbf{D}\left(v_{\text {eff }}-v_{\text {cst }}\right) \underline{\overline{\mathbf{S}}}$ is far smaller than the one of the convective term. Therefore the stability is indeed enforced by the CFL limit.

The implicit part is solved by a generalized block LU decomposition, using a standard fractional-step method with pressure correction which may be preconditioned by various algorithms.

\section{FILTERING}

As spectral elements offer high accuracy for the flow at hand, we construct the filters using two spectral techniques. The first one is a nodal filter acting in physical space on the nodal velocity components (and pressure) to stabilize the computations. The second method is designed as a modal filter and is carried out element-wise in spectral space and corresponds to the convolution kernel of the LES filtering.

\subsection{Nodal Filter}

The nodal filter is due to Mullen and Fischer [14] and is adequately suited to parallel spectral element computation. Introducing $h_{N, j}, j=$ $0, \ldots, N$ the set of Lagrange-Legendre interpolant polynomials of degree $N$ on the GLL grid nodes $\xi_{N, k}, k=0, \ldots, N$, the rectangular matrix operator $I_{N}^{M}$ of size $(M+1) \times(N+1)$ is such that

$$
\left(I_{N}^{M}\right)_{i j}=h_{N, j}\left(\xi_{M, i}\right) .
$$

Therefore, the matrix operator of order $N-1$

$$
\Pi_{N-1}=I_{N-1}^{N} I_{N}^{N-1}
$$


interpolates on the GLL grid of degree $N-1$ a function defined on the GLL grid of degree $N$ and transfers it back to the original grid. By this process, one can show that one gets rid of the highest modes of the polynomial representation. The $1 D$ filter is given by the relation

$$
\bar{v}=\left[\alpha \Pi_{N-1}+(1-\alpha) I_{N}^{N}\right] v .
$$

The LES version of the filter sets $\alpha=1$ and is given by

$$
\bar{v}=I_{M}^{N} I_{N}^{M} v,
$$

where $M$ is equal to $N-2$ or $N-3$. The extension to three-dimensional problems results easily from the matrix tensor product properties of the filter.

\subsection{Modal Filter}

The variable $v$ aside its Lagrange-Legendre representation may also be approximated by a modal basis that was first proposed in the $p$ version of the finite elements. That basis was used by Boyd [2] as a filter technique and is built up on the reference parent element as

$$
\phi_{0}=\frac{1-\xi}{2}, \phi_{1}=\frac{1+\xi}{2}, \phi_{k}=L_{k}(\xi)-L_{k-2}(\xi), \quad 2 \leqslant k \leqslant N .
$$

The one-to-one correspondence between the Lagrangian basis and the $p$ representation yields

$$
v\left(\xi_{i}\right)=\sum_{k=0}^{N} \hat{v}_{k} \phi_{k}\left(\xi_{i}\right),
$$

which in matrix notation reads

$$
\mathbf{v}=\boldsymbol{\Phi} \hat{\mathbf{v}} .
$$

The filter operation is performed in spectral space through a diagonal matrix $\mathbf{T}$ with components such that

$$
T_{k}=\frac{1}{\left(1+\left(k / k_{c}\right)^{2}\right)},
$$

where the cut-off value $k_{c}$ corresponds to $T_{k}=1 / 2$. The entire filtering process for a $1 D$ problem is given by

$$
\overline{\mathbf{v}}=G \star \mathbf{v}=\boldsymbol{\Phi} \mathbf{T} \boldsymbol{\Phi}^{-1} \mathbf{v} .
$$


The extension to three-dimensional is trivial by the matrix tensor product properties.

The transfer function $T_{k}$ of Eq. (23) corresponds to a standard lowpass filter required when using any spectral method [2].

\subsection{The Filter Length}

For a $1 D$ problem using the SEM, a common choice [7] of filter length is

$$
\Delta=\frac{s}{p},
$$

where $s$ is the element size and $p$ the highest polynomial degree in the spectral decomposition Eq. (21) that is the closest to the frequency $k_{c}$

$$
p=k, \quad \text { such that } \inf _{k}\left(\left|k-k_{c}\right|\right)<1 / 2, \quad k=0, \ldots, N .
$$

We notice that the filter length decreases when the element is refined. The $3 D$ formula for rectilinear elements is

$$
\Delta(x, y, z)=\left(\Delta_{1}(x) \Delta_{2}(y) \Delta_{3}(z)\right)^{1 / 3}=\left(\frac{s_{1}}{p_{1}} \frac{s_{2}}{p_{2}} \frac{s_{3}}{p_{3}}\right)^{1 / 3} .
$$

\section{THE LID-DRIVEN CUBICAL CAVITY PROBLEM}

The lid-driven cavity presents although the geometry is simple, complex physical phenomena. As in this case, we have no homogeneous direction, the presence of side walls confining the full flow modifies the flow patterns and the route to turbulence. For the description of the physics among the abundant literature, we refer the reader to Koseff and Street [8], Jordan and Ragab [5], Leriche and Gavrilakis [10], and Shankar and Deshpande [16].

Figure $1 \mathrm{~b}$ shows the cubical cavity. The flow motion is induced by the top lid that moves in the $x$-direction with a constant unit velocity $U_{0}=$ 1. The Reynolds number is consequently $\operatorname{Re}=U_{0} 2 h / v$. We will essentially address the case of the flow at $\operatorname{Re}=12,000$. The kinetic energy is provided to the flow by the shear stress at the top lid through viscous diffusion. The amplitude of the Reynolds stress below the lid is negligible indicating that the flow under the lid is mainly laminar but transient. The momentum transfer from the lid induces a region of strong pressure in the upper corner of the downstream wall as the flow, mainly horizontal prior the corner, 
(a)

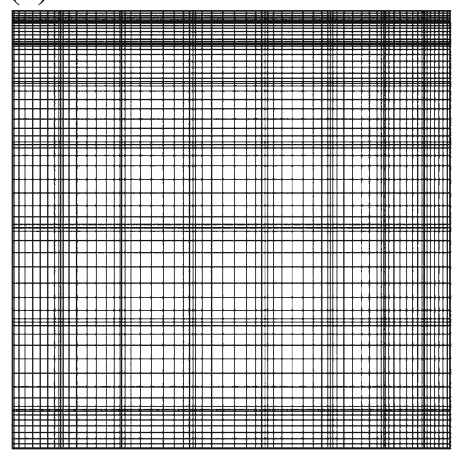

(b)

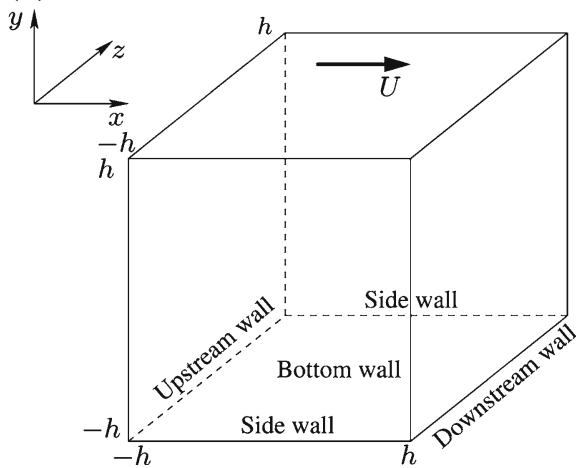

Fig. 1. (a) Spectral element grid in the mid-plane $z=0$ and (b) Sketch of the geometry of the lid-driven cubical cavity.

has to change direction and moves vertically downwards. This sharp turn dissipates energy in that region. Along the downstream wall the plunging flow behaves like a jet with a variable thickness. Near the symmetry plane the jet thickness is reduced while it increases away from this plane. This jet, laminar and unsteady at the very beginning, separates from the cavity wall at mid-height and grows as two elliptical jets on both sides of the symmetry plane. They hit the bottom wall where they produce turbulence. This turbulence is convected away by the main central vortex towards the upstream wall where the flow slows down and relaminarizes during the fluid rise.

In order to resolve the boundary layers along the lid and the downstream wall, the spectral elements are unevenly distributed as can be seen in Fig. 1a. The spatial discretization has $E_{x}=E_{y}=E_{z}=8$ elements in the three space directions with $N_{x}=N_{y}=N_{z}=8$ polynomial degree. The spectral element calculation has two times less points per space direction than the DNS of Leriche-Gavrilakis [10] who employed a $129^{3}$ Chebyshev discretization.

As far as the velocity imposed on the lid is concerned, the unit velocity induces severe discontinuities along the top edges. In order to remove these defects we use a high degree polynomial as Leriche and Gavrilakis did

$$
u=\left[1-(x / h)^{18}\right]^{2}\left[1-(z / h)^{18}\right]^{2}, \quad v=w=0 .
$$

No-slip conditions are applied to the other walls.

Both nodal and modal filters were used in our LES computations; the former with $M=N-2$ to stabilize the velocity field at each time step 
and the latter with $k_{c}=N-2$ to filter the highest modes in the Legendre space. The computations are particularly sensitive to the values of $M$ and $k_{c}$; smaller values will affect spectral convergence whereas higher values will have very little effect on the smallest scales of the problem.

The dynamic constant $C_{\mathrm{d}}$ produced has high values in the regions of high velocity gradients. Its maximum value fluctuates around 0.25 with locally some negative values which are eliminated by clipping.

The time step is chosen as $\Delta t=10^{-3}$ and the complete simulation comprises 201,000 iterations leading to a total effective simulation time of 201 time units. The reference results are the DNS data of Leriche [9] and the experimental ones from Koseff and Street [8], corresponding to 1,000 and 145.5 time units respectively. In the cavity flow, the average is obtained by time averaging.

The results of an under-resolved DNS performed on the coarse grid made of $65^{3}$ grid points and designed for the LES are presented on Fig. 2b. These results are compared to the same results for the LES (Fig. 2a) and for the DNS (Fig. 2c). A qualitative comparison of the three sets of mean-velocity contours for $\langle U\rangle$ and $\langle V\rangle$ allows to conclude to the failure of the under-resolved DNS to reproduce the physics involved in this problem. As expected the resolved-scale spectrum is smaller than

(a)

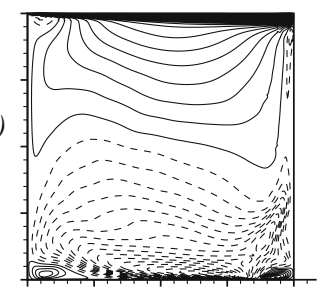

(ii)

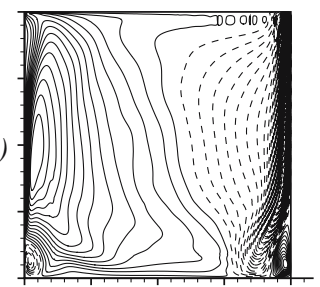

(b)
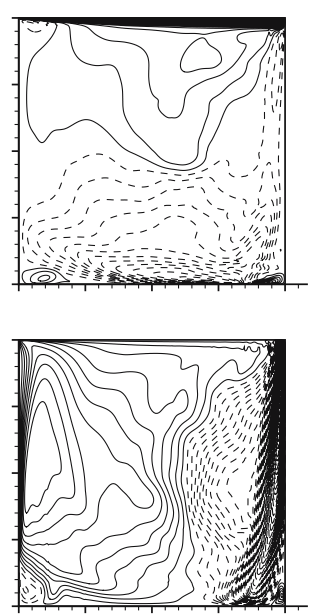

(c)
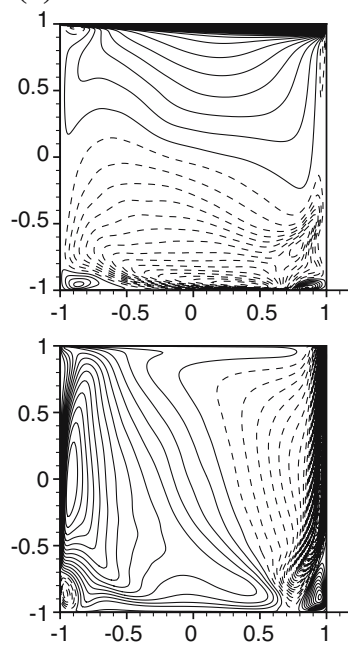

Fig. 2. Contours of the $x$-component of the mean velocity field $\langle U\rangle$ (row $(i)$ ) and of the $y$-component $\langle V\rangle$ (row (ii)) in the mid-plane $z=0$ for column (a) the LES, column (b) the under-resolved DNS, column (c) the DNS from Leriche and Gavrilakis [10]. 

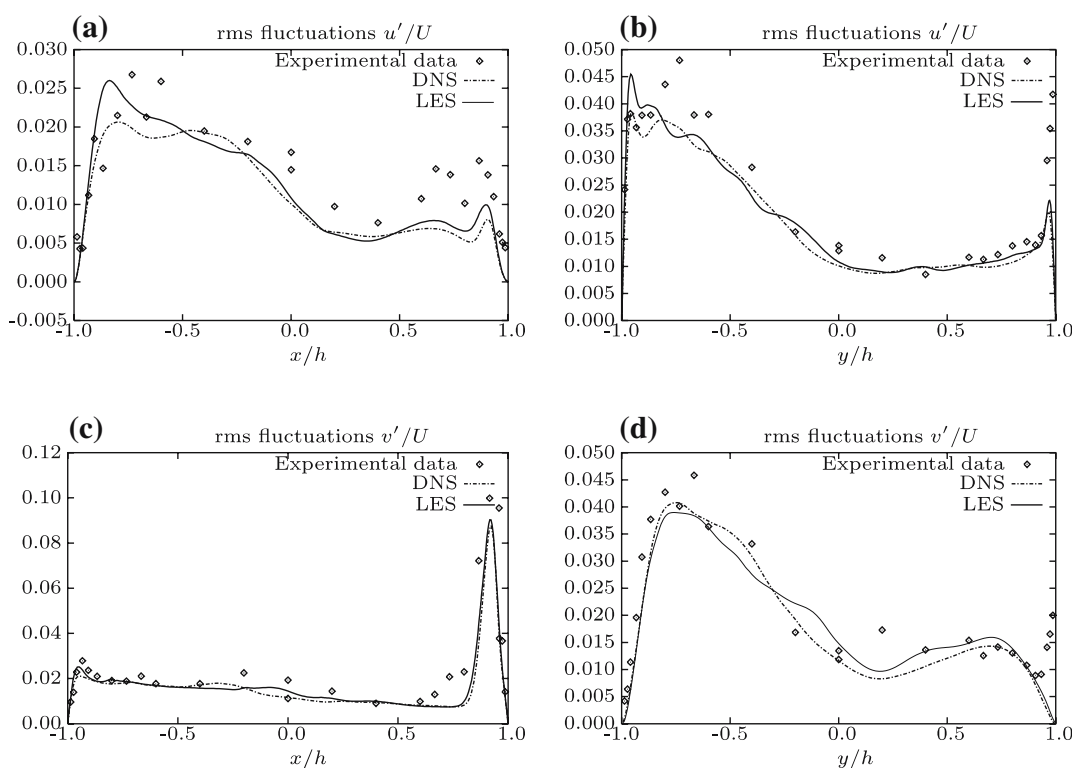

Fig. 3. Profiles of the rms fluctuations $u^{\prime}$ ((a) and (b)), $v^{\prime}$ ((c) and (d)) in the mid-plane $z=0$, along the lines $x=0$ ((b) and (d)) and $y=0$ ((a) and (c)), for the LES, the DNS from Leriche and Gavrilakis [10] and the experimental data from Koseff and Street [8].

the full-scale spectrum for this turbulent flow and therefore necessitates to model the under-resolved scales as it is done in our LES (Fig. 2a).

From a more quantitative point-of-view, the $x$-component $u^{\prime}$ and the $y$-component $v^{\prime}$ of the rms fluctuations of the velocity field have been computed along two $1 D$ lines $x=0$ and $y=0$ in the mid-plane $z=0$. Results for the LES are shown on Fig. 3 together with the DNS and experimental results, proving the excellent performance of our SGS modelling. The differences of the three sets of data in terms of peak amplitude observed can be explained by the three different ensemble averaging used as proved by Leriche [9]. A larger ensemble averaging tends to reduce the amplitude of the different peaks.

\section{CONCLUSION AND FUTURE STUDIES}

A LES of the three-dimensional lid-driven cubical cavity flow using the SEM has been presented. The treatment of the subgrid-scales relies on a dynamic model for the eddy viscosity. This LES has been carried out on a parallel architecture with a relatively coarse grid and numerical results 
appeared to be extremely close to the DNS and experimental results available in the literature. Moreover the results of the under-resolved DNS on the coarse grid are far from providing any insight into the physics of the problem.

Our next goal is to perform the same LES but with other treatments of the subgrid-scales and determine the most efficient model when the simulation are based on the SEM.

\section{ACKNOWLEDGMENTS}

This research is being partially funded by a Swiss National Science Fundation Grant (No. 200020-101707), whose support is gratefully acknowledged.

\section{REFERENCES}

1. Blackburn, H. M., and Schmidt, S. (2003). Spectral element filtering techniques for large eddy simulation with dynamic estimation. J. Comput. Phys. 186, 610-629.

2. Boyd, J. P. (1998). Two comments on filtering (artificial viscosity) for Chebyshev and Legendre spectral and spectral element methods: Preserving boundary conditions and interpretation of the filter as a diffusion. J. Comput. Phys. 143, 283-288.

3. Deville, M. O., Fischer, P. F., and Mund, E. H. (2002). High-Order Methods for Incompressible Fluid Flow, Cambridge Monographs on Applied and Computational Mathematics, Cambridge University Press, Cambridge.

4. Germano, M., Piomelli, U., Moin, P., and Cabot, W. H. (1991). A dynamic subgrid-scale eddy viscosity model. Phys. Fluids A. 3, 1760-1793.

5. Jordan, S. A., and Ragab, S. A. (1994). On the unsteady and turbulent characteristics of the three-dimensional shear-driven cavity flow. J. Fluids Eng. 116, 439-449.

6. Karamanos, G. S., Sherwin, S. J., and Morrison, J. F. (1999). Large eddy simulation using unstructured spectral/hp elements. In Knight, D., and Sakell, L. (eds.), Recent Advances in DNS and LES. Kluwer.

7. Karamanos, G. S., and Karniadakis, G. E. (2000). A spectral vanishing viscosity method for Large-Eddy simulations. J. Comput. Phys. 163, 22-50.

8. Koseff, J. R., and Street, R. L. (1984). The lid-driven cavity flow: a synthesis of qualitative and quantitative observations. J. Fluids Eng. 106, 390-398.

9. Leriche, E. (1999). Direct numerical simulation of lid-driven cavity flow by a Chebyshev spectral method. Ph. D. Thesis no. 1932. Swiss Federal Institute of Technology, Lausanne.

10. Leriche, E., and Gavrilakis, S. (1999). Direct numerical simulation of the flow in the liddriven cubical cavity. Phys. Fluids. 12, 1363-1376.

11. Lesieur, M., and Métais, O. (1996). New trends in large-eddy simulations of turbulence. Ann. Rev. Fluid Mech. 28, 45-82.

12. Lilly, D. K. (1992). A proposed modification of the Germano subgrid-scale closure method. Phys. Fluids A. 4, 633-635. 
13. Maday, Y., and Patera, A. T. (1989). Spectral element methods for the NavierStokes equations. In Noor, A. K., and Oden, J. T. (eds.), State-of-the-Art Surveys in Computational Mechanics, ASME, New York, pp. 71-143.

14. Mullen, J. S., and Fischer, P. F. (1999). Filtering techniques for complex geometry fluid flows. Comm. Numer. Meth. Eng. 15, 9-18.

15. Sagaut, P. (2003). Large Eddy Simulation for Incompressible Flows - An Introduction, 2nd edn, Springer, Berlin.

16. Shankar, P. N., and Desphande, M. D. (2000). Fluid mechanics in the driven cavity. Annu. Rev. Fluid Mech. 32, 93-136.

17. Smagorinsky, J. S. (1963). General circulation experiments with the primitive equations. Month. Weather Rev. 91, 99-164. 